\title{
SINGLE CENTRE CLINICAL EXPERIENCE WITH FIDAXOMICIN IN THE TREATMENT OF CLOSTRIDIUM DIFFICILE INFECTION IN SLOVAKIA
}

\author{
Martin Novotný1, Pavol Jarčuška1', Laura Gombošová3, Ján Hockicko', Ivana Hockicková1, Alena Rovňáková1, \\ Ondrej Zahornacký', Ivan Schréter ${ }^{1}$, Erik Dorko², Kvetoslava Rimárová2 \\ 'Department of Infectology and Travel Medicine, Faculty of Medicine, Pavol Jozef Šafárik University in Košice and Louis Pasteur University \\ Hospital, Košice, Slovak Republic \\ 2Department of Public Health and Hygiene, Faculty of Medicine, Pavol Jozef Šafárik University in Košice, Košice, Slovak Republic \\ ${ }^{3} 1$ st Department of Internal Medicine, Faculty of Medicine, Pavol Jozef Šafárik University in Košice and Louis Pasteur University Hospital, \\ Košice, Slovak Republic
}

\section{SUMMARY}

Objective: Clostridium difficile infection (CDI) has become one of the most common causes of hospital-acquired infections. Fidaxomicin is one of the latest antibiotics used in the treatment of $\mathrm{CDI}$, however, treatment cost affects recommendations for its use in several countries. We have analysed the treatment of our patients with CDI, treated by fidaxomicin since it was introduced to the market in 2018 and became available in the second biggest Slovak hospital, University Hospital of L. Pasteur. Our aim was to determine efficacy and safety of fidaxomicin in the treatment of CDI in Slovak patients.

Methods: We reviewed all courses of fidaxomicin use in our hospital $(n=60)$. Fidaxomicin was used for first recurrence $(12$ times $)$, second recurrence (4 times), third recurrence (2 times), and fifth recurrence (1 patient). 41 patients received fidaxomicin first-line.

Results: Success of fidaxomicin treatment was recorded at $86.7 \%$ within the whole cohort. In the recurrent Clostridium difficile infection (rCDI) subgroup, fidaxomicin was $63 \%$ effective with three patients dying (15.7\%) and two patients developing subsequent rCDI. During the duration of the study, 6 patients in total died. Only one of three patients, with three or more recurrences of CDI, had no further presentations after eight weeks of completion of treatment.

Conclusions: The biggest benefit from fidaxomicin treatment was shown in a cohort of patients with primary CDI infection demonstrating a low recurrence rate and significant reduction of fidaxomicin effectiveness in preventing a recurrence when treating patients with multiple rCDI.

Key words: Clostridium difficile infection, fidaxomicin treatment, recurrence

Address for correspondence: K. Rimárová, Department of Public Health and Hygiene, Faculty of Medicine, Pavol Jozef Šafárik University in Košice, Šrobárova 2, 04180 Košice, Slovak Republic. E-mail: kvetoslava.rimarova@upjs.sk

https://doi.org/10.21101/cejph.a5476

\section{INTRODUCTION}

Antibiotics displayed their dark side at the end of the last century. As well as the growth of multi-resistant hospital acquired bacterial species, clostridium enterocolitis was a newly acquired illness difficult to manage. Clostridium difficile infection has become one of the most common causes of hospital-acquired infections. Antibiotic therapy causes alterations to the intestinal microbial composition, enabling Clostridium difficile colonization and consecutive toxin production leading to disruption of the colonic epithelial cells. Clinical symptoms of CDI range from mild diarrhoea to potentially life-threatening conditions like pseudomembranous colitis or toxic megacolon (1). Clostridium difficile, with its ability to produce spores and colonise the gastrointestinal tract, became a major public health problem.
On top of the increased incidence of Clostridium difficile infection (CDI) there was an increase in the recurrence of CDI (rCDI). Recurrent CDI is associated with significantly increased mortality. CDI incidence rate depends on the local prevalence of CDI and it increases with inpatient's length of stay, age and immunodeficiency (2).

Based on Slovak guidelines, severe illness is defined if at least one risk factor is present in a patient with $\mathrm{CDI}$ :

- Age over 65

- Concurrent treatment with broad spectrum antibiotics

- Antibiotic treatment in past 3 months

- Prolonged admission (longer than 3 weeks)

- Previous or current stay in a carer institution

- Complex underlying illness (immunocompromised patients, cancer patients and transplant patients) 
- Abdominal surgery, colorectal carcinoma, chronic inflammatory bowel disease (mainly Crohn's disease)

- Treatment with $\mathrm{pH}$ altering medications (antacids, H2 antagonists, proton pump inhibitors)

- Nasogastric or PEG feeding

- Hypoalbuminemia

- Chronic renal insufficiency (clearance of creatinine lower than $0.5 \mathrm{~mL} / \mathrm{s}$ )

- Diabetes mellitus

- Fever over $38.5^{\circ} \mathrm{C}$

- Evidence of severe colitis.

The prevalence of asymptomatic Clostridium difficile colonization varies depending on the number of hosts, pathogens, and environmental factors; asymptomatic colonization may be underestimated as routine stool culture is not practical in a clinical setting. Individuals who are colonized by the organism have the potential to contribute to its transmission in healthcare settings (3). Public Health Authority of the Slovak Republic estimate that $7-11 \%$ of inpatients, $5-7 \%$ of people using social facilities or nursing homes and about $2 \%$ of outpatients are colonised with Clostridium difficile (2).

The incidence of CDI increased by tenfold in the last five years. There were 263, 922, 1,121, 1,435 and 1,942 cases in 2013, 2014, 2015 and 2016 respectively. In 2017, 2,604 CDI cases were reported (morbidity $47.9 / 100,000$ ), this is a $25.4 \%$ increase compared with 2016 . CDI was reported in every region of Slovakia. The highest incidence was reported in Bratislava (90.5) and the lowest in Banská Bystrica (28.6). National CDI incidence based on age group are as follows: less than one year $=41,1-4=37,5-9=9,10-14=2,15-19=15$, $20-24=13,25-34=56,35-44=65,45-54=205,55-64=295$, $65+=1,866$. The majority of these cases were hospital acquired $(1,935-74.3 \%)$. (4)

First line antibiotics are losing efficacy in the treatment of CDI (metronidazole), or increased selective pressure on bacterial resistance (vancomycin). This highlighted a need for a new antibiotic. The answer was fidaxomicin; a bacteriocidic, macrolide antibiotic with a narrow spectrum of effectiveness - primarily for Clostridium difficile. It is one of the most recently discovered antibiotics on the market, which is associated with higher cost when compared to other commonly used antibiotics (5).

A large number of specialist clinics recommend treatment with fidaxomicin for severe cases of CDI or rCDI. Its cost however, leads to sparse utilisation of this antibiotic. Australasian Society for Infectious Diseases recommends fidaxomicin as first line treatment for the second recurrence of CDI or second line refractory CDI. This recommendation is based on a low cost-effectiveness of fidaxomicin as a first-line treatment for severe CDI (6). However, there is some evidence suggesting its cost effectiveness when assessing prevention of recurrence and complications (7).

Slovak guidelines for the diagnosis and management of CDI follows recent recommendations from specialised clinics and despite its cost, can be used for the benefit of not only the patient but also public finances. Vancomycin continues to be first line treatment, but fidaxomicin can be used for treatment of recurrent CDI as well as treatment of severe CDI of patients with high risk of recurrence (2).

\section{MATERIALS AND METHODS}

We collected data from 60 adult patients, aged 18 and over, using a single centre retrospective cohort study from 01/01/2013 to $01 / 01 / 2018$. University Hospital of Louis Pasteur in Košice, Slovakia (UHLP) has 1,356 beds, 15,000 admissions a year and is the second biggest hospital in Slovakia.

Diagnosis of CDI: diagnosis was confirmed using $\mathrm{RIDA}^{\circledR} \mathrm{QUICK}$ Clostridium difficile Toxin A/B Control, RIDA ${ }^{\circledR}$ QUICK Clostridium difficile GDH Control test performed on patients with diarrhoea or a finding of pseudomembranous enterocolitis during colonoscopy.

Data collected included basic demographic data (age, sex and duration of admission), place of primary infection of CDI, severity of CDI, number of recurrences, previous antibiotic treatment and previous CDI treatment, recurrence following fidaxomicin, adverse effects of fidaxomicin treatment, immunity status, preexisting oncological diagnosis and chemotherapy prior treatment.

Patients were divided into 4 groups based on severity of CDI (8). (Table 1)

CDI risk was determined using ATLAS and CSI ( $C$. difficile severity index) scoring system (Table 2 and 3). ATLAS - Bedside Scoring System is composed of 5 components: Age, Temperature, Leukocytes, Albumin, and Systemic antibiotics (2).

Recurrence of CDI was diagnosed as CDI recurrence less than 8 weeks from initial CDI Infection. Successful treatment was defined as:

Table 1. CDI classification based on disease severity (8)

\begin{tabular}{|l|l|l|}
\hline Category & Signs & Associated risk factors \\
\hline Mild to moderate & $\begin{array}{l}\text { Diarrhea without systemic signs of infection, } \\
\text { WBC count }<15,000 \text { cells/mL, serum creatinine }<1.5 \text { times } \\
\text { baseline }\end{array}$ & $\begin{array}{l}\text { Antibiotic use, previous hospitalization, longer duration of } \\
\text { hospitalization, use of PPI, receipt of chemotherapy, chronic } \\
\text { kidney disease }\end{array}$ \\
\hline Severe & $\begin{array}{l}\text { Systemic signs of infection, and/or WBC count }>15,000 \text { cells/mL, } \\
\text { or serum creatinine }>1.5 \text { times the premorbid level }\end{array}$ & Advanced age, infection with BI/NAP1/027 strain \\
\hline Severe, complicated & $\begin{array}{l}\text { Systemic signs of infection including hypotension, ileus, } \\
\text { or megacolon }\end{array}$ & $\begin{array}{l}\text { See above, plus recent surgery, history of IBD, and intravenous } \\
\text { immunoglobulin treatment }\end{array}$ \\
\hline Reccurent & $\begin{array}{l}\text { Recurrence within } 8 \text { weeks of successfully completing treatment } \\
\text { for CDI }\end{array}$ & $\begin{array}{l}\text { Patient age }>65 \text { years concomitant antibiotic use, presence of } \\
\text { significant comorbidities, concomitant use of PPI, and increased } \\
\text { initial disease severity }\end{array}$ \\
\hline
\end{tabular}

WBC - white blood cells; PPI - proton pump inhibitors; IBD - intestine bowel disease 
Table 2. ATLAS - prediction of mortality scoring system for patients with CDI (2)

\begin{tabular}{|l|c|c|c|}
\hline Parameter & 0 points & 1 point & 2 points \\
\hline Age & $<60$ & $60-79$ & $>80$ \\
\hline Temperature & $<37.5^{\circ} \mathrm{C}$ & $37.5-38.5^{\circ} \mathrm{C}$ & $>38.6^{\circ} \mathrm{C}$ \\
\hline WBC count (cells/mL) & $<16,000$ & $16,000-25,000$ & $>25,000$ \\
\hline Albumin $(\mathrm{g} / \mathrm{L})$ & $>35$ & $26-35$ & $<25$ \\
\hline Systemic ATB treatment & no & & yes \\
\hline
\end{tabular}

WBC - white blood cells; ATB - antibiotics

Scores above 6 suggested increased mortality

Table 3. CSI - prediction of mortality scoring system for patients with CDI (2)

\begin{tabular}{|l|c|c|}
\hline Parameter & 0 points & 1 point \\
\hline Creatinine & $<1.5$ times normal & $>1.5$ times normal \\
\hline WBC count $($ cellls $/ \mathrm{mL})$ & $<20,000$ & $>20,000$ \\
\hline Albumin $(\mathrm{g} / \mathrm{L})$ & $<30$ & $>30$ \\
\hline
\end{tabular}

WBC - white blood cells

Scores above 2 suggested increased mortality

1. Reduced frequency of bowel motion or consistency of bowel motion without mucus on day 3 .

2. No evidence of severe colitis.

3. No signs of organ failure/septic shock (2).

\section{RESULTS}

During the duration of the study, 4,819 stool samples were tested of which 784 tested positive for CDI (Table 4).

During the observed period there were 206 CDI related admissions to the Department of Infectology and Travel Medicine of UHLP. Ten patients were readmitted twice and two patients required three readmissions related to CDI. 50 patients were treated with fidaxomicin ( $200 \mathrm{mg}$ twice daily, orally). Ten other patients were also treated with fidaxomicin ( $200 \mathrm{mg}$ twice daily, orally) on different wards of University Hospital of Louis Pasteur.

Fidaxomicin was used for first recurrence (12 times), second recurrence (4 times), third recurrence ( 2 times) and fifth recurrence (1 patient). 41 patients received fidaxomicin first-line. The average length of admission was 10.2 days.

The study cohort consisted of 60 patients, 35 females and 25 males respectively. Average age of patients admitted was 72.45 years (range 35 to 94 years). When sorting patients depending on risk groups, 12 patients were in the less than 60 year old group, 23 patients were in the 60-80 year old group and 25 patients were over 80 years old. Eight patients died; two of them died as a result of illness not related to CDI (cerebrovascular accident, endocarditis prior to $\mathrm{CDI}$ ).
All patients included in this study had severe CDI based on the Slovak guidance for assessment and management of CDI. Depending on ATLAS score severity; 15 patients had moderate CDI, 33 patients had severe CDI and 12 patients had severe complicated CDI (Table 5). CSI and ATLAS scores of patients treated with fidaxomicin are listed in Table 6 and 7 . First presentation of CDI was treated in 41 patients based on high risk of recurrence. 19 patients developed recurrence.

Fidaxomicin was well tolerated and no adverse reactions were observed in the duration of the study. Treatment failed in eight patients treated by fidaxomicin; six patients progressed into septic shock and subsequently died. Two patients treated with fidoxamicin developed rCDI within eight weeks of first treatment. Success of treatment was recorded at $86.7 \%$ within the whole cohort. In rCDI subgroup fidaxomicin was $63 \%$ effective with three patients dying (15.7\%) and two patients developed subsequent rCDI.

\section{DISCUSSION}

Based on the most recent in vivo and in vitro studies, fidaxomicin is a very effective antibiotic. One of the biggest advantages is its narrow spectrum of efficacy and pharmacokinetics that only sparsely affects gastrointestinal flora and other anatomical systems. The biggest disadvantage is its high cost when compared to vancomycin $(9,10)$.

Median effectiveness in other studies varies between 50\% (11) and $92.5 \%(9,13)$. Our study showed $86.7 \%$ effectiveness in the whole cohort without assessing recurrence. In patients treated for CDI and less than two rCDI, fidoxamicin was $90.2 \%$ effective in treatment and $100 \%$ effective in preventing further recurrence. Timing of treatment seems to make the biggest impact of its effectiveness. If treating patients with first presentation of CDI or first or second recurrence of CDI, fidaxomicin is shown to be superior to vancomycin. Fidoxamicin loses its superiority to vancomycin when treating patients with multiple recurrences (over three) $(11,12)$.

The cost effectiveness of fidaxomicin has to be viewed in a broad setting. Higher cost may predispose the use of this anti-

Table 4. Summary of tests performed in UHLP performed between 01/01/2013 to 01/01/2018

\begin{tabular}{|l|c|c|c|c|c|}
\hline & $\mathbf{2 0 1 3}$ & $\mathbf{2 0 1 4}$ & $\mathbf{2 0 1 5}$ & $\mathbf{2 0 1 6}$ & $\mathbf{2 0 1 7}$ \\
\hline Number of tests & 601 & 750 & 984 & 1,117 & 1,367 \\
\hline CDI positive results & $118(20 \%)$ & $130(17 \%)$ & $179(18 \%)$ & $164(15 \%)$ & $193(14 \%)$ \\
\hline
\end{tabular}


Table 5. Patient distribution based on disease severity $(N=60)$

\begin{tabular}{|l|c|c|c|}
\hline CDI disease category & $\mathbf{n}$ & $\begin{array}{c}\text { 1st line } \\
\text { treatment }\end{array}$ & Recurrences \\
\hline Mild to moderate & 15 & 9 & 6 \\
\hline Severe & 33 & 21 & 12 \\
\hline Severe complicated & 12 & 11 & 1 \\
\hline \multicolumn{2}{|l|}{ Recurrent } \\
*from whole group and every severity \\
$19^{*}$
\end{tabular}

Table 6. Patient distribution based on CSI scoring system $(N=60)$

\begin{tabular}{|l|c|c|c|}
\hline CSI & $\mathbf{n}$ & $\begin{array}{c}\text { 1st line } \\
\text { treatment }\end{array}$ & Reccurences \\
\hline Missing data & 1 & 1 & 0 \\
\hline 0 point & 10 & 9 & 1 \\
\hline 1 points & 23 & 15 & 8 \\
\hline 2 points & 14 & 8 & 6 \\
\hline 3 points & 12 & 8 & 4 \\
\hline
\end{tabular}

Table 7. Patient risk stratification based on ATLAS score $(N=60)$

\begin{tabular}{|l|c|c|c|}
\hline ATLAS score & $\mathbf{n}$ & $\begin{array}{c}\text { 1st line } \\
\text { treatment }\end{array}$ & Recurrences \\
\hline Missing data & 2 & 1 & 1 \\
\hline $0-1$ point & 0 & 0 & 0 \\
\hline 2 points & 4 & 1 & 3 \\
\hline 3 points & 5 & 4 & 1 \\
\hline 4 points & 13 & 7 & 6 \\
\hline 5 points & 13 & 10 & 3 \\
\hline 6 points & 9 & 7 & 2 \\
\hline 7 points & 6 & 5 & 1 \\
\hline 8 points & 3 & 2 & 1 \\
\hline 9 points & 3 & 3 & 0 \\
\hline 10 points & 2 & 1 & 1 \\
\hline
\end{tabular}

biotic in only the most severe cases. In our cohort, patients with mild presentation of CDI but high risk of recurrence, were also treated with fidoxamicin. Our patients treated with fidaxomicin for first presentation as well as first or second recurrence of CDI did not develop further recurrence of CDI. It has to be noted that our result may be skewed due to the small number of patients, when compared to larger studies $(14,15)$.

Patients with multiple rCDI had an increased risk of further recurrence, $66.7 \%$. Only one of three patients, with three or more recurrences of CDI, had no further presentations after eight weeks of treatment. The remaining two patients developed a recurrence within 28 days. It is important to note that in this small group no deaths were observed despite severe CDI. Similar findings are already documented in other studies with a small number of patients $(11,12,16)$.

The phrase 'Prevention is better than cure' is commonly used in medical circles and it can be applied in CDI management. Preventing Clostridium difficile transmission and infection continues to be a challenge. The epidemiology of this infection is changing, but basic rules still apply. To reduce the incidence of CDI, three principles must apply: prevention of CDI, surveillance of CDI (through mandatory reporting) and prevention of further transmission of CDI. Primary prevention measures are aimed at antibiotic restriction policies, early patient mobilisation and the reduction of length of hospital stay. Key components for CDI surveillance are appropriate case definitions of CDI and standardized CDI diagnostics with a focus on high risk departments. Basic measures to prevent transmission include isolation of every case of CDI (especially while diarrhoea is present) and environmental disinfection of CDI. Hand hygiene is a crucial recommendation for preventing and controlling the transmission of $\mathrm{CDI}$, including the use of disposable gloves and handwashing with soap and water (2).

To summarise, our results correlate with recently published studies showing the potential use of fidaxomicin in the prevention of recurrences of CDI when treating patients with first presentation and up to two recurrences of CDI. Just as demonstrated by Enoch et al. (11), we too have recorded a significant reduction of its effectiveness in preventing a recurrence when treating patients with multiple rCDI. This finding needs to be studied further due to the limited number of patients. Despite its cost, prevention of recurrence leads to increased savings of public finances by preventing recurrent infections in patients with milder forms of CDI when risk factors for recurrence are present.

\section{Acknowledgements}

This publication is the result of the project implementation: Medicínsky univerzitný vedecký park v Košiciach (MediPark, Košice - Fáza II., code ITMS2014+ 313011D103 supported by the Operational Programme Research \& Innovation, funded by the ERDF and Grants VEGA No. 1/0198/13, 1/0011/14 and 1/0941/16 of the Ministry of Education, Science, Research and Sport of the Slovak Republic We thank also Directory board of Louis Pasteur University Hospital in Košice for disclosure of patients information.

\section{Conflict of Interests}

None declared

\section{REFERENCES}

1. Schäffler H, Breitrück A, Clostridium difficile - from colonization to infection. Front Microbiol. 2018;9:646. doi: 10.3389/ fmicb.2018.00646.

2. Jarčuška P, Bátovský M, Drgoňa L', Lišková A, Holečková K. Guidelines for diagnosis and treatment of colitis caused by Clostridium difficile. Via Practica. 2015;12(S1):4-11. (In Slovak.)

3. Furuya-Kanamori L, Marquess J, Yakob L, Riley TV, Paterson DL, Foster NF, et al. (2015). Asymptomatic Clostridium difficile colonization: epidemiology and clinical implications. BMC Infect Dis. 2015;15:516. doi: 10.1186/s12879-015-1258-4.

4. Public Health Authority of the Slovak Republic. Annual report [Internet]. Public Health Authority of the Slovak Republic [cited 2019 Jan 9]. Available from: http://www.uvzsr.sk/index.php?option=com_content\&view= category\&layout $=\mathrm{blog} \& \mathrm{id}=25 \&$ Itemid $=34$. (In Slovak.) 
5. Zhanel GG, Walkty AJ, Karlowsky JA. Fidaxomicin. A novel agent for the treatment of Clostridium difficile infection. Can J Infect Dis Med Microbiol. 2015 Nov-Dec;26(6):305-12.

6. Fehér C, Mensa J. A comparison of current guidelines of five international societies on Clostridium difficile infection management. Infect Dis Ther. 2016 Sep;5(3):207-30.

7. Nathwani D, Cornely OA, Van Engen AK, Odufowora-Sita O, Retsa P, Odeyemi IA. Cost-effectiveness analysis of fidaxomicin versus vancomycin in Clostridium difficile infection. J Antimicrob Chemother. 2014 Nov;69(11):2901-12.

8. Bagdasarian N, Rao K, Malani PN. Diagnosis and treatment of Clostridium difficile in adults: a systematic review. JAMA. 2015 Jan;313(4): 398-408.

9. Louie TJ, Cannon K, Byrne B, Emery J, Ward L, Eyben M, et al. Fidaxomicin preserves the intestinal microbiome during and after treatment of Clostridium difficile infection (CDI) and reduces both toxin reexpression and recurrence of CDI. Clin Infect Dis. 2012 Aug;55(2):S132-42.

10. Sears P, Crook DW, Louie TJ, Miller MA, Weiss K. Fidaxomicin attains high fecal concentrations with minimal plasma concentrations following oral administration in patients with Clostridium difficile infection. Clin Infect Dis. 2012 Aug; 55(2):S116-20.

11. Enoch D, Santos R, Phillips C, Micallef C, Murphy M, Aliyu S, et al. Real-world use of fidaxomicin in a large UK tertiary hospital:
How effective is it for treating recurrent disease? J Hosp Infect. 2018 Oct;100(2):142-6.

12. Orenstein R. Fidaxomicin failures in recurrent Clostridium difficile infection: a problem of timing. Clin Infect Dis. 2012 Aug;55(4):613-4.

13. Cornely OA, Crook DW, Esposito R, Poirier A, Somero MS, Weiss K, et al.; OPT-80-004 Clinical Study Group. Fidaxomicin versus vancomycin for infection with Clostridium difficile in Europe, Canada, and the USA: A double-blind, non-inferiority, randomised controlled trial. Lancet Infect Dis. 2012 Apr;12(4):281-9.

14. Lee C, Louie TJ, Weiss K, Valiquette L, Gerson M, Arnott W, et al. Fidaxomicin versus Vancomycin in the treatment of Clostridium difficile infection: Canadian outcomes. Can J Infect Dis Med Microbiol. 2016;2016:8048757. doi: 10.1155/2016/8048757.

15. Vaishnavi C. Fidaxomicin - the new drug for Clostridium difficile infection. Indian J Med Res. 2015 Apr;141(4):398-407.

16. Vargo CA, Bauer KA, Mangino JE, Johnston JE, Goff DA. An antimicrobial stewardship program's real-world experience with fidaxomicin for treatment of Clostridium difficile infection: a case series. Pharmacotherapy. 2014 Sep;34(9):901-9.

Received July 4, 2018

Accepted in revised form December 28, 2018 